\title{
X-ray diffraction and extended $x$-ray absorption fine-structure characterization of nonspherical crystallographic grains in iron thin films
}

\author{
F. Jiménez-Villacorta, A. Muñoz-Martín, ${ }^{\text {a) }}$ and C. Prieto ${ }^{\text {b) }}$ \\ Instituto de Ciencia de Materiales de Madrid, Consejo Superior de Investigaciones Cientificas, \\ Cantoblanco, 28049 Madrid, Spain
}

(Received 24 May 2004; accepted 5 September 2004)

\begin{abstract}
The characterization of iron thin films grown at different substrate temperatures has been performed by $\mathrm{x}$-ray diffraction (XRD) and extended $\mathrm{x}$-ray absorption fine structure (EXAFS). The film growing of iron at low temperatures provides an excellent system to test the results obtained from both techniques because the crystallographic grains present a variation of size and shape as a function of the growing temperature. In both cases, the shape of the particle must be taken into account to calculate their size. The comparison gives a very good agreement when appropriate models are used, showing the reasons for possible differences between the results obtained from a more simple XRD and EXAFS analysis applied to columnar growth systems. (C) 2004 American Institute of Physics.
\end{abstract} [DOI: $10.1063 / 1.1810636]$

\section{INTRODUCTION}

In the last decade, the study of ultrafine magnetic particles has shown a great development because of their attractive applications in technological and materials research. ${ }^{1}$ The evolution in the field of nanoscience has led to methods providing a precise structural study of nanoparticles and nanostructures to become very important. This is due to the special magnetic properties of small particles, different in comparison with the bulk. These singular properties are directly affected by size, shape, and distribution effects, so accurate methods to control their formation and to measure them are required.

There are several techniques to obtain the average particle size of materials. The most employed of these is $\mathrm{x}$-ray diffraction (XRD), which allows calculation of the coherence length, being an estimation of the particle size present at the samples. In the standard Bragg-Brentano configuration, also called $\theta-2 \theta$, incidence and detection angles vary simultaneously. When the transferred momentum between the incident and the scattered beam coincides with a reciprocallattice vector (which is perpendicular to a family of lattice planes), a diffraction peak will appear. For a polycrystalline thin film the diffractogram is similar to those obtained from powder materials. ${ }^{2}$ So the transferred momentum that gives diffraction peaks is always perpendicular to the film. The coherence length along the direction of the transferred momentum is obtained by measuring the width of the diffraction peak and by using the Scherrer formula. This method is commonly used to determine the size of the crystalline grains. ${ }^{3}$

Another technique that has been recently used to estimate the size of nanoparticles is the extended x-ray absorption fine structure (EXAFS). ${ }^{4}$ It is based on the oscillatory behavior of the x-ray absorption coefficient starting somewhat after the absorption edge of an inner atomic level. Con-

\footnotetext{
${ }^{a)}$ Present address: Centro de Microanálisis de Materiales, Universidad Autónoma de Madrid, Cantoblanco, 28049 Madrid, Spain

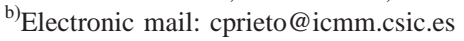

versely to the diffraction techniques, it is based on the shortrange order ${ }^{5}$ and provides information about the local coordination environment of a selected atom, including crystal structure, distances between atoms, as well as coordination number $(\mathrm{CN})$. With this information, it is possible to estimate the size of a particle by calculating the reduction of the average $\mathrm{CN}$ due to the finite-size effect. For that purpose, it should be considered that atoms at the cluster surface have lost some of their neighbors. In order to find the relationship between size and the lost of average coordination number, a geometrical shape model must be introduced. ${ }^{6}$ In most cases, a spherical $^{3,6-9}$ or a hemispherical ${ }^{9-11}$ shape model introducing the relationship between the decreasing size of the particle and the loss of average coordination number was proposed.

A comparison between XRD and EXAFS grain-size results shows that for particles with nanometric sizes and spherical shapes, both techniques provide similar values. ${ }^{3,7}$ But, for a polycrystalline thin film in which grains can have nonspherical shapes (as it, generally, occurs for columnar growth of films), the results of both techniques are not in agreement. Generally, this discrepancy provides smaller size values when the $\mathrm{x}$-ray absorption technique is used. Moreover, it seems that for broad size distributions, the spherical shape grain models are weighted by the smaller size range when EXAFS results are interpreted, while for XRD analysis, size appears to be closer to the bigger size range of the distribution. ${ }^{3}$

In this paper, we present XRD and EXAFS data of a set of iron thin-film samples prepared at different temperatures by sputtering. This system has the peculiarity to provide a variation of the crystallographic grain size with the preparation temperature that has an important influence on their magnetic properties. ${ }^{12,13}$ Moreover, their poor microstructural texture allows the use of a method based on XRD measurements to measure the crystallographic grain dimensions (these can have nonspherical shapes) along both relevant directions, the normal to the surface and the one contained in 
the film plane. The method is adequate for this type of systems due to the frequent columnar growth of thin films. Additionally, columnar growth exhibits their more compact crystallographic plane parallel to the surface (which is called texture). These effects are common for thin films prepared by sputtering. Under these assumptions, we propose a way of estimating the grain shape by XRD, based on measuring different coherence lengths along different directions with respect to the film and using the Scherrer formula. It should be remarked that these measurements are possible because films do not have a very high degree of texture.

For EXAFS analysis, we will introduce a model that takes into account not only the loss of coordination number of atoms at the surface of the grain but also its shape. Additionally, linear polarization effects are considered in order to reproduce the experiment where the relative position between the columns of the film and the polarization of the $\mathrm{X}$-ray beam is known.

\section{EXPERIMENT}

Iron thin films were grown by sputtering on naturally oxidized $\mathrm{Si}(100)$ substrates using a dc-operated 2-in. planar magnetron source (from Angstrom Sciences). Base pressure was in the high-vacuum range $\left(10^{-7} \mathrm{mbar}\right)$ and Ar pressure during sputtering deposition was $5.0 \times 10^{-3} \mathrm{mbar}$. The deposition rate was about $1 \mathrm{~nm} / \mathrm{min}$; the power applied to the magnetron was about $1 \mathrm{~W}$, and both were kept constant in the process. With all these growth parameters, we obtain iron layers with a thickness of about $500 \AA$. The experimental setup has the possibility of keeping the substrate temperature constant during deposition, by using a cold finger attached to a home-modified Oxford CF-100 continuous flow cryostat placed inside the vacuum chamber. ${ }^{12,13}$ This device is controlled by an Oxford ITC-502 temperature controller, which allows us to set the substrate temperature with a precision of $0.1 \mathrm{~K}$. Moreover, in order to deposit a cap layer, there exists the possibility of changing sputtering targets, maintaining the sample at high-vacuum pressure range.

For this work, two sets of samples were prepared: the first one was covered with a gold layer in order to prevent natural oxidation and the second one was oxidized in situ at room temperature in the vacuum chamber with an oxygen partial pressure of $5 \times 10^{-1}$ mbar for $1 \mathrm{~h}$, and subsequently capped with the gold layer in order to prevent further oxidations. The substrate temperature during iron deposition was varied from 300 to $170 \mathrm{~K}$.

$\mathrm{X}$-ray diffraction experiments were performed in a twocircle $\mathrm{x}$-ray diffractometer (D8 Brucker-AXS), by using the standard $\theta-2 \theta$ (also called Bragg-Brentano) geometry. X-ray absorption have been carried out at LURE (DCI, XAS-13 beamline) with an electron-beam energy of $1.85 \mathrm{GeV}$ and average current of $250 \mathrm{~mA}$. Data of the Fe K-edge were obtained maintaining the sample at $100 \mathrm{~K}$ by using a fixed exit monochromator with two flat $\mathrm{Si}(111)$ crystals; detection was made by collecting the total electron yield on a biased electrode in a He atmosphere sample chamber. ${ }^{14}$ The experimental geometry was chosen in such a manner that x-ray polarization vector was contained in the sample surface.
EXAFS analysis was performed by using the VIPER program. ${ }^{15}$ Oscillations were obtained after removing the background by a cubic spline fitting polynomial, and EXAFS signal $[\chi(k)]$ was obtained by normalizing the magnitude of the oscillations to the edge jump. The resulting data were transformed into a function of the photoelectron wave number, $k$.

The pseudoradial distribution function around iron atoms has been calculated by Fourier transforming the $k$-weighted EXAFS signal $[k \cdot \chi(k)]$ multiplied previously by a Hanning window. For average CN estimation and comparative study, the back Fourier transform of the first peak (corresponding to the first and second distances in the $b c c-\mathrm{Fe}$ ) was fitted to the well-known expression ${ }^{5}$

$$
\begin{aligned}
\chi(k)= & S_{0}^{2} \sum_{j} \frac{\left(3 N_{j} \cos ^{2} \theta_{j}\right)}{k R_{j}^{2}} e^{\left(-2 k^{2} \sigma_{j}^{2}\right)} e^{\left[-2 R_{j} / \lambda(k)\right]} f_{j}(k) \\
& \times \sin \left[2 k R_{j}+\Phi_{j}(k)\right] .
\end{aligned}
$$

Equation (1) describes the EXAFS oscillations for a Gaussian distribution of $N_{j}$ atoms at mean distances $R_{j}$ around the absorbing atom considering single-scattering and plane-wave approximation. $S_{0}^{2}$ is an intrinsic loss factor, $N_{j}$ is the average coordination number for the Gaussian distribution of distances centered at the $R_{j}$ value, $\sigma_{j}$ is the DebyeWaller (DW) factor, and $\phi_{j}(k)=2 \delta(k)+\gamma_{j}(k)$ is the phase shift, $\delta(k)$ and $\gamma_{j}(k)$ being the central- and backscatteringatom phase shifts, respectively. $f_{j}(k)$ is the magnitude of the backscattering amplitude of the $j$ th-neighbor atom, and $\lambda$ is the mean free path of the photoelectron traveling from the absorbing atom to the backscatterer in the $j$ th shell and in the lifetime of the core hole. Finally, $\theta_{j}$ is the angle between the absorbing-backscatterer bond and the polarization vector of the incoming $\mathrm{x}$-ray beam.

In the fit, the amplitude and phase functions have been calculated by using the FEFF code version 6.01, reported by Rehr. ${ }^{16}$ The amplitude loss factor $\left(S_{0}^{2}\right)$ was obtained by fitting the spectrum of a Fe foil used as a reference.

\section{RESULTS AND DISCUSSION}

\section{A. X-ray diffraction}

In order to estimate the grain size, the coherence length is calculated from the full width at half maximum (FWHM) of iron 110-bcc diffraction peak and then using the Scherrer formula. For thin films, the Bragg-Brentano geometry maximizes signal for textures normal to the surface because of specular position of the sample, with respect to the incident and diffracted beam.

Figure 1 shows the "rocking" curves of some samples, the detector has been fixed at the iron (110)-bcc diffraction angle $\left(2 \theta=44.6^{\circ}\right)$. The intensity of those rocking curves spread over $\pm 20^{\circ}$ from the maximum, which indicates that it is possible to find (110)-bcc planes at any direction in the film. The maximum value is found at $\theta=22.3^{\circ}$, corresponding to one-half of the (110) Bragg angle, indicating a poor texture normal to the film plane. This fact allows us to perform diffraction measurements with the transferred momen- 


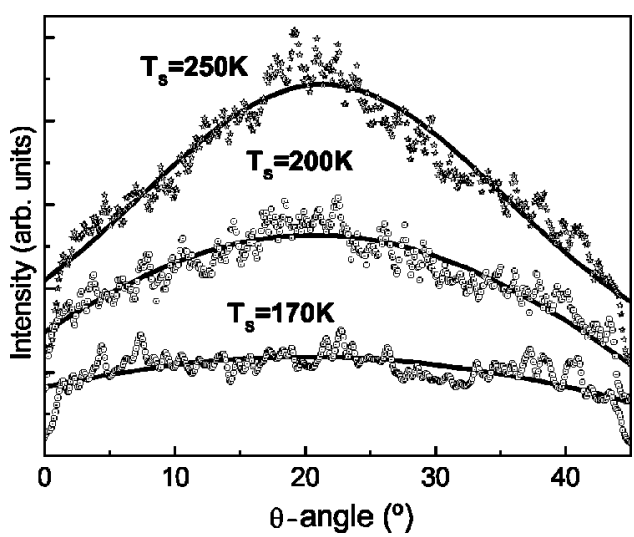

FIG. 1. Rocking curves of in situ oxidized samples.

tum $\mathbf{K}$, responsible for diffraction, along directions different from the perpendicular one, and subsequently to evaluate coherence length in those directions.

Figure 2(a) shows the experimental arrangement used in order to obtain the grain sizes along different directions. Crystallographic grains are considered as spheroids, taking into account the columnar growth of these films. As it is shown, standard XRD implies a $\theta-2 \theta$ specular geometry, where $\theta$ is the incidence angle over the surface; the coupling between $\theta$ and $2 \theta$ makes the coherence length obtained a measurement of the grain size along the normal to the film. For nonspecular geometries, the direction of the transferred momentum scans different angles from the normal to the film, and the obtained lengths can be used to estimate the lateral size of the columns. By considering the coherence length dependence on the incident angle, it is possible to obtain the dimensions of the grains along the "out-of-plane" direction (perpendicular to the film), and also to evaluate the
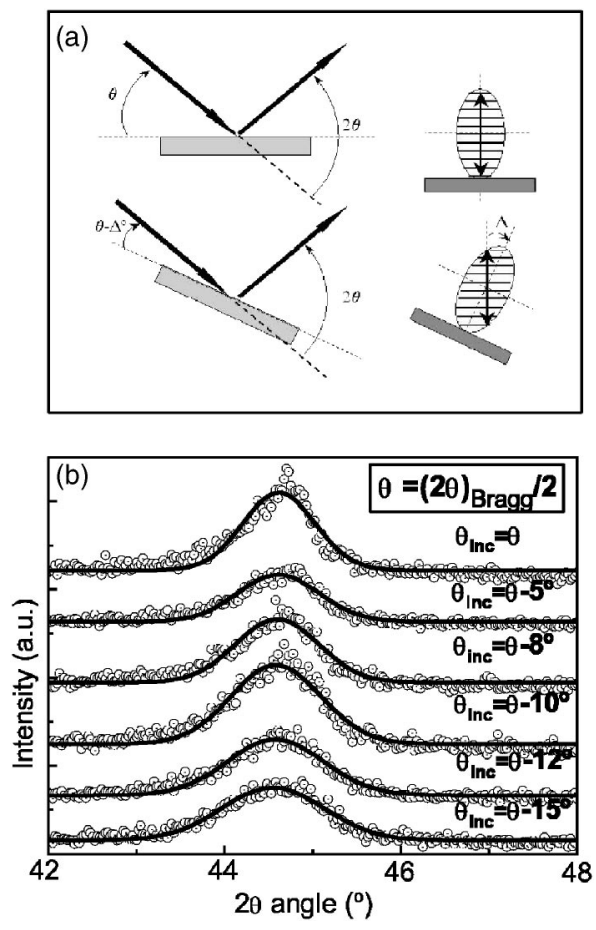

FIG. 2. (a) Experimental arrangement for XRD measurements and (b) evolution of (110)-bcc iron peak obtained for different incident angles. dimension along the "in-plane" one (parallel to the film). These data provide the information needed to consider crystallographic grains as having spherical shape or, otherwise, to obtain their eccentricity for a spheroidal grain model.

Figure 2(b) shows the evolution of the (110)-bcc iron diffraction peak at different incident angles. For every peak, it is possible to calculate their coherence length by using the Scherrer expression, $\left\langle L_{c}\right\rangle=0.94 \lambda /[B(2 \theta) \times \cos \theta]$, where $\lambda$ is the wavelength of incident $\mathrm{x}$ ray $(\lambda=1.54 \AA)$ and $B(2 \theta)$ is the FWHM of the diffraction peak.

According to what is observed in Fig. 2(b), the obtained coherence lengths show a clear decrease when the incident angle diminishes. In order to obtain the dimensions of the grains, a geometrical model should be proposed. For this purpose, we consider a spheroidal prolate shape to determine the in-plane and the out-of-plane dimensions of the grain. Figure 3 represents the experimental coherence lengths obtained at different angles. Incidence angle is referred to as $\theta_{\text {inc }}=\frac{1}{2}(2 \theta)_{\text {Bragg }}-\Delta$ in order to show the deviation from the specular conditions. Solid lines show the variation of the ellipse diameter, calculated as a function of the angle that runs from the largest diameter to the smallest one. If the spheroidal grains form the film as Fig. 2 depicted, this angle coincides with the formed between the perpendicular to the film and the contained in the film plane, which is the $\Delta$ angle. Calculations are obtained from the ellipse equation and, for every sample, fitting is made by varying the long and short axes values of this equation.
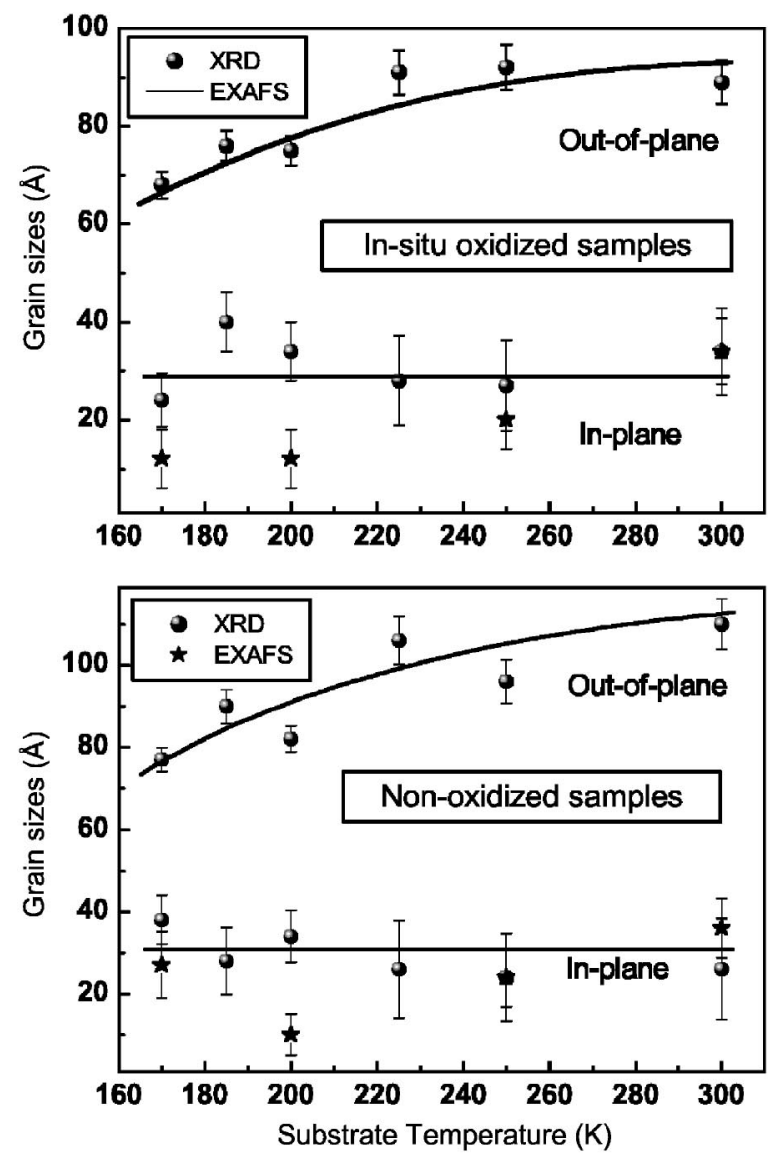

FIG. 4. Grain sizes estimated from the XRD and EXAFS. (a) In situ oxidized samples and (b) gold-capped samples. 


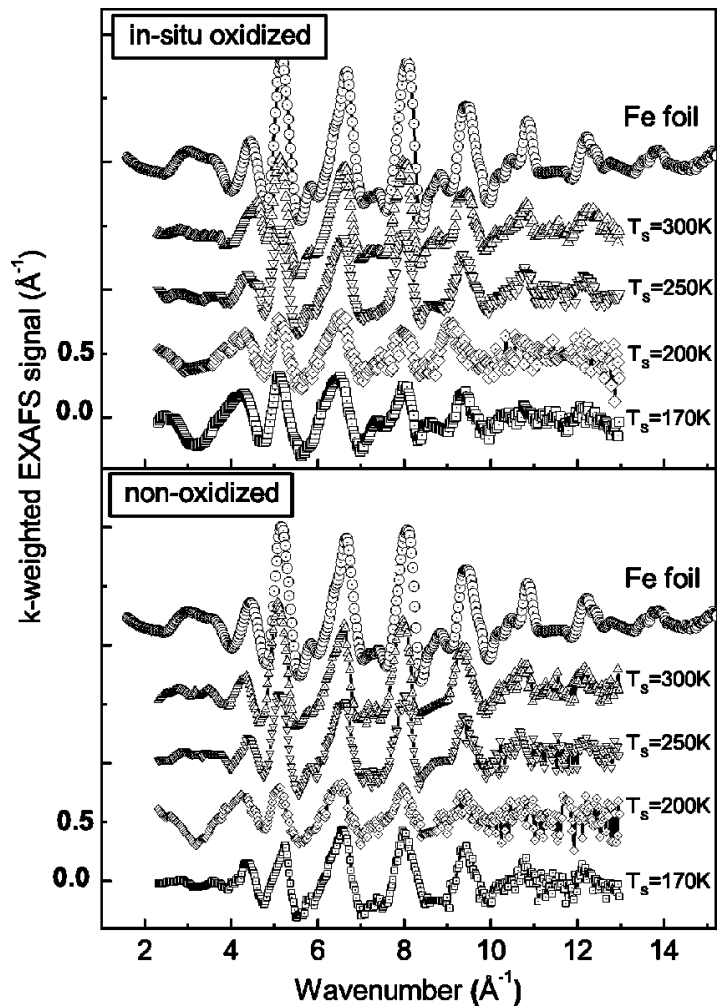

FIG. 5. $k$-weighted EXAFS spectra of iron after atomic background substraction, for samples prepared at different substrate temperatures $\left(T_{S}=300\right.$, 250, 200, and $170 \mathrm{~K}$ ). (a) In situ oxidized samples and (b) gold-capped samples.

The results obtained using this method are shown in Fig. 4 for both series of samples. At first glance, it is possible to see that out-of-plane sizes decrease as the substrate temperature diminishes. However, the in-plane sizes remain almost constant for all of them. Moreover, it can be observed that all the samples have grains with elongated shape, which is not affected by oxidation, although it produces a slight decrease in the out-of-plane sizes, in agreement with a preferential oxidation on top of the columns.

\section{B. X-ray absorption spectroscopy}

Figures 5 and 6 show the EXAFS signals and their respective Fourier transforms of the two sets of samples. Fou-

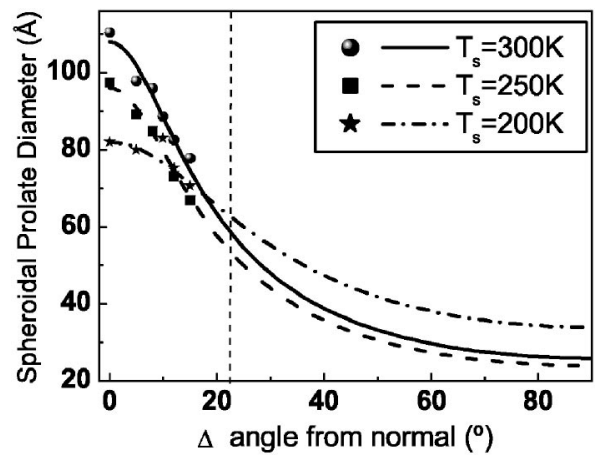

FIG. 3. Experimental diffraction data of samples (symbols) and fit by spheroidal model (solid lines). In the nonspecular arrangement, there is no possibility of measuring diffraction at an angle, $\Delta$, larger than one-half of the Bragg angle $\left(\Delta=22.3^{\circ}\right)$.

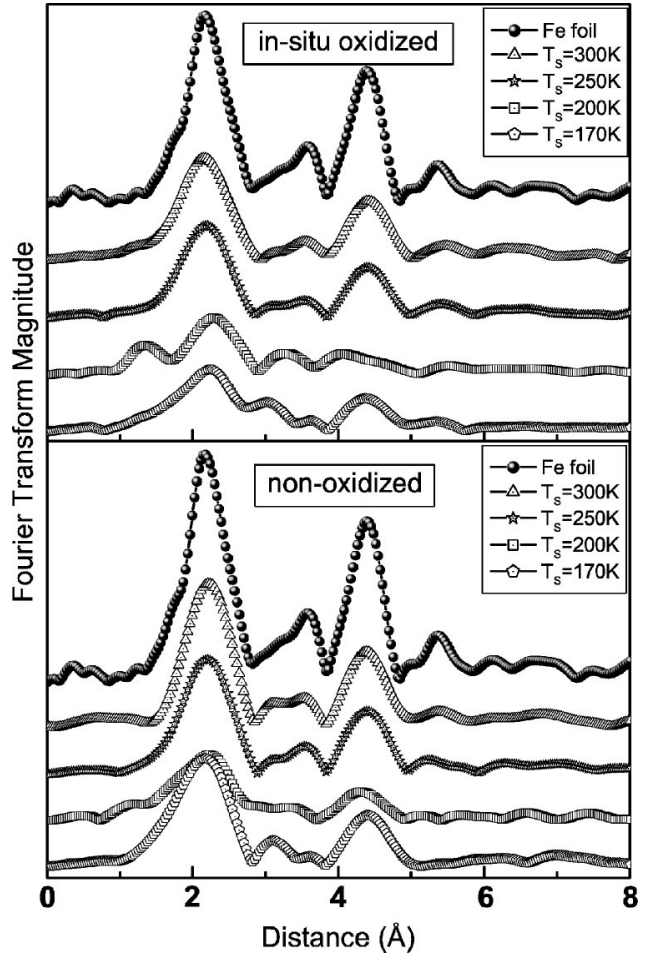

FIG. 6. Fourier transform of EXAFS spectra of samples of Fig. 5. (a) In situ oxidized samples and (b) gold-capped samples.

rier transform magnitudes are related to coordination shells of the absorbing atom. Their amplitude evolution with the preparation substrate temperature gives a qualitative description of the reduction in the number of neighbors, with respect to the reference foil. That indicates a loss of nearest neighbors due to finite-size effect. For atoms at the surface of a grain, the $\mathrm{CN}$ is near one-half of the corresponding for a bulk atom. For very small grain sizes, this effect reduces the measured coordination number, due to the more important statistical surface to volume weight in the estimation of the average CN. Nevertheless, there is an anomaly for the 200-K-prepared sample of both series. This may be correlated with the film microstructure that provides a higher oxidation that give rise to an anomalous magnetic behavior. ${ }^{13}$ This higher oxidation, with respect to the other samples of the same series, has been qualitatively detected by x-ray appearance near-edge structure spectroscopy. ${ }^{17}$

Table I displays the first coordination shell parameters, obtained after the EXAFS data analysis. The results reveal a clear reduction of the average coordination number when the substrate temperature decreases. Moreover, the distances between atoms remain constant and in agreement with the parameters of iron $b c c$ lattice and no appreciable increases are observed at the Debye-Waller factor.

As it has been reported above, the average $\mathrm{CN}$ is an accurate parameter to estimate the grain size. Taking into account the fact that the studied samples have grains with columnar shape, we will develop a model to calculate the average coordination number for a geometrical figure in agreement with the XRD study. For that purpose, we consider cylindrical shape particles, because, compared to spheroidal forms, cylinders provide an easy calculation of the 
TABLE I. Summary of distances $\left(R_{j}\right)$, number of neighbors $\left(N_{j}\right)$, and Debye-Waller factors $\left(\sigma_{j}\right)$ obtained from fitting EXAFS signal, for first and second coordination distances.

\begin{tabular}{|c|c|c|c|c|}
\hline Sample & $T_{S}(\mathrm{~K})$ & $N_{j}$ & $R_{j}(\AA)$ & $\sigma_{j}\left(\AA^{-1}\right)$ \\
\hline \multirow[t]{2}{*}{ Fe foil } & \multirow[t]{2}{*}{300} & 8.0 & 2.48 & 0.071 \\
\hline & & 6.0 & 2.84 & 0.071 \\
\hline \multirow{8}{*}{$\begin{array}{c}\text { In situ } \\
\text { oxidized }\end{array}$} & \multirow[t]{2}{*}{300} & 6.7 & 2.49 & 0.075 \\
\hline & & 4.1 & 2.85 & 0.075 \\
\hline & \multirow[t]{2}{*}{250} & 5.7 & 2.49 & 0.070 \\
\hline & & 3.1 & 2.85 & 0.070 \\
\hline & \multirow[t]{2}{*}{200} & 3.3 & 2.54 & 0.070 \\
\hline & & 2.3 & 2.89 & 0.070 \\
\hline & \multirow[t]{2}{*}{170} & 4.3 & 2.50 & 0.074 \\
\hline & & 1.7 & 2.87 & 0.074 \\
\hline \multirow[t]{8}{*}{ Nonoxidized } & \multirow[t]{2}{*}{300} & 6.6 & 2.50 & 0.072 \\
\hline & & 4.4 & 2.88 & 0.072 \\
\hline & \multirow[t]{2}{*}{250} & 5.6 & 2.49 & 0.072 \\
\hline & & 3.8 & 2.86 & 0.072 \\
\hline & \multirow[t]{2}{*}{200} & 3.5 & 2.51 & 0.072 \\
\hline & & 1.5 & 2.88 & 0.072 \\
\hline & \multirow[t]{2}{*}{170} & 6.2 & 2.49 & 0.073 \\
\hline & & 3.7 & 2.84 & 0.073 \\
\hline
\end{tabular}

number of atoms at the surface and at the inner volume and there is no appreciable difference between both geometrical forms. The method is similar to that proposed by Borowski ${ }^{7}$ and Calvin et al., ${ }^{3}$ but we have changed the spherical to a cylindrical shape to model the crystalline grains of these films. This model is explained as follows: let us suppose a cylinder of radius $R$ and height $h$, as it is shown in Fig. 7, then, we define the radius of the coordination shell as $d$. Atoms placed at every part have different contributions to the average $\mathrm{CN}$. For $b c c$ iron, we have taken $d=2.68 \AA$, which is the average between the first and the second neighbors distances, as we note that both distances are too close to distinguish them and the first coordination sphere is formed by both of them.

For this cylinder, we can distinguish four parts, whose volumetric fraction, with respect to the total volume, is given in Table II: the inner part, the lateral surface, the two bases, and the two edges. Subsequently, in order to calculate the average $\mathrm{CN}$, each one of the external volumetric fractions must be multiplied by two reduction factors: the first one due

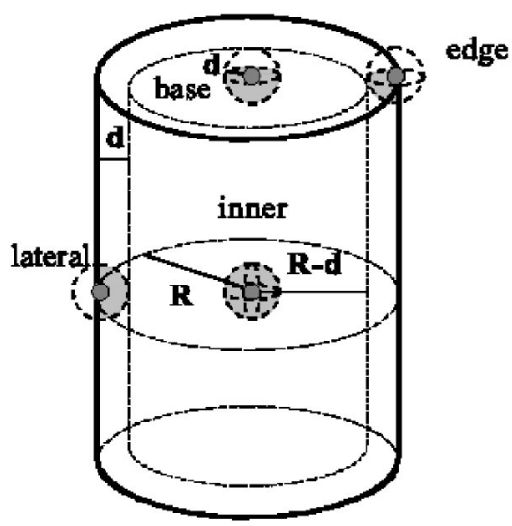

FIG. 7. Cylindrical shape model distinguishing the four regions analyzed.
TABLE II. Mathematical expression for the volumetric fraction of the four considered parts of the cylinder (see Fig. 7), neighbors loss factor and polarization factor.

\begin{tabular}{cccc}
\hline \hline & Volumetric fraction & $\begin{array}{c}\text { Neighbors } \\
\text { occupancy }\end{array}$ & $\begin{array}{c}\text { Polarization } \\
\text { factor }\end{array}$ \\
\hline Inside & $I n=(R-d)^{2}(h-2 d) / R^{2} h$ & $N_{I n}=1$ & $P_{I n}=1$ \\
Lateral & $L a=2(R-d) d(h-2 d) / R^{2} h$ & $N_{L a}=7 / 14$ & $P_{L a}=4 / 7$ \\
Bases & $B a=2(R-d)^{2} d / R^{2} h$ & $N_{B a}=10 / 14$ & $P_{B a}=11 / 10$ \\
Edges & $E d=2(R-d) d^{2} / R^{2} h$ & $N_{E d}=5 / 14$ & $P_{E d}=4 / 5$ \\
\hline \hline
\end{tabular}

to surface loss of neighbors $\left(N_{\text {surf }}\right)$ and the second one due to the polarization effect $\left(P_{\text {surf }}\right)$. This second reduction term must take into account the relative position between the $\mathrm{x}$-ray polarization and the axis of the cylinder. The x-ray polarization effect has been used to study the variation of the nearest neighbors distance along different directions in $\mathrm{Co} / \mathrm{Cu}$ multilayers ${ }^{18}$ and in magnetic ultrathin films. ${ }^{19}$ Then, the expression for the total average $\mathrm{CN}$ as a function of the cylindrical column dimensions is

$$
\mathrm{ACN}=\langle\widetilde{N}\rangle=I n+N_{L a} P_{L a} L a+2 N_{B a} P_{B a} B a+2 N_{E d} P_{E d} E d .
$$

In order to reproduce the experimental conditions, the polarization reduction factor has been calculated for an incoming beam whose polarization was contained in the film plane. (In Table II, the polarization factor is given for cylinders with their axis aligned along the perpendicular to the $\mathrm{x}$-ray polarization vector.) After substitution, Eq. (2) yields to the more compact expression

$$
\langle\tilde{N}\rangle=1-\frac{10}{7}\left(\frac{d}{R}\right)+\frac{3}{7}\left(\frac{d}{R}\right)^{2}-\frac{3}{7 \alpha}\left(\frac{d}{R}\right)\left[1-\left(\frac{d}{R}\right)\right]^{2},
$$

where $\alpha=h / R$.

Figure 8 shows the averaged $\mathrm{CN}$ dependence with the dimensions of the columnar grain. An average cylinder dimension has been defined in order to compare the dependence with the obtained for a spherical grain. These calculations permit us to obtain the cylinder radius that fit the loss of coordination number obtained by EXAFS spectroscopy. It

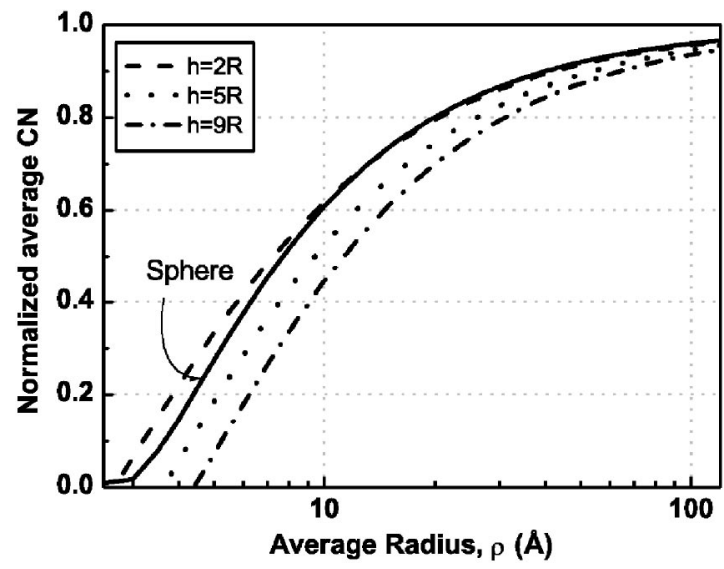

FIG. 8. Normalized average $\mathrm{CN}$ as a function of cylinder radius and height. The average dimension parameter is defined as $\rho=\left(h R^{2}\right)^{1 / 3}$ in order to compare with the spherical shape particles. 
can be observed that the increase of the height/radius ratio $(h / R)$ makes a more significant loss in the average $\mathrm{CN}$ than that observed for a cylinder with $h=2 R$, which is the most similar to a sphere.

The dimensions obtained by EXAFS results have been plotted in Fig. 4 in order to be compared with those obtained by XRD. For this columnar growth-studied system, the EXAFS-obtained dimensions are related with the radius (or diameter) of the columns, which corresponds to the in-plane size. As it can be observed, the agreement is excellent for both series of samples, where the in-plane dimensions obtained by XRD are very similar to those obtained by EXAFS after applying the proposed grain modelization. Additionally, this agreement is in accordance with the validity of both techniques to determine the grain size, but when both of them are used in a suitable way. As a final remark, it can be concluded that XRD size evaluation is generally determined by the largest dimension of the grains and EXAFS size evaluation is more sensitive to the smallest grain dimension and, for an ordered microstructure, it is influenced by the polarization measurement direction.

\section{CONCLUSIONS}

We have studied the crystalline size and shape behavior in iron thin films prepared by sputtering at very low temperatures. Two slightly different systems (due to their oxidation) have been studied. We use XRD and EXAFS data to estimate the relevant dimensions of the columnar crystals in these films. The size and shape of the thin-film grains depend on the substrate temperature during deposition. Columnar growth gives elongated crystal grains and less sharp forms for growth at lower temperature.

The XRD analysis was possible due to the poor texture of these films. Dimensions of the grains are obtained, giving a nearly constant value of approximately $30 \AA$ for the inplane size and an out-of-plane size that changed from 110 to $70 \AA$ with decreasing temperature from 300 to $170 \mathrm{~K}$. This has been possible after applying a geometrical method in which we have considered the grains having an elongated shape.

The EXAFS analysis has been made taking into account the shape provided by the XRD results. The EXAFS results show a significant reduction of the $\mathrm{CN}$ in thin films, with respect to the bulk iron. A cylindrical model according to elongated shape of crystallographic grains has been used to obtain their characteristic size. The EXAFS-derived dimension was in good agreement with those obtained for the inplane size derived from XRD analysis, corresponding to the diameter of the columnar grains. This work shows that both XRD and EXAFS techniques are able to provide useful information about the shape and average dimensions of crystallites in samples with nanometric grains.

\section{ACKNOWLEDGMENTS}

The authors would like to thank Professor A. Traverse from LURE for his suggestions in the EXAFS data analysis. The authors also acknowledge the staff in charge of the DCI storage ring of LURE for the beam time allocation, as well as for the European Large Installation program. This work has been supported by Spanish DGICYT under Contract No. MAT2003-01880.

${ }^{1}$ I.M.L. Billas, A. Chatelain, and W.A. de Heer, Science 265, 1682 (1994). ${ }^{2} \mathrm{C}$. Prieto and A. de Andrés, in Láminas Delgadas y Recubrimientos: Preparación, Propiedades y Aplicaciones, edited by J.M. Albella (CSIC, Madrid, 2003).

${ }^{3}$ S. Calvin, M.M. Miller, R. Goswami, S.F. Cheng, S.P. Mulvaney, L.J. Whitman, and V.G. Harris, J. Appl. Phys. 94, 778 (2003).

${ }^{4}$ A. Traverse, New J. Chem. 22, 677 (1998).

${ }^{5}$ D.C. Koninsberger and R. Prins, X-Ray Absorption: Principles, Applications, Techniques of EXAFS, SEXAFS and XANES, (Wiley, New York, 1988).

${ }^{6}$ R.B. Greegor and F.W. Lytle, J. Catal. 63, 476 (1980).

${ }^{7}$ M. Borowski, J. Phys. IV (France) 7, C2-259 (1997).

${ }^{8}$ I. Arcon, A. Tuel, A. Kodre, G. Martín, and A. Barbier, J. Synchrotron Radiat. 8, 575 (2001).

${ }^{9}$ A.I. Frenkel, C.W. Hills, and R.G. Nuzzo, J. Phys. Chem. 105, 12689 (2001).

${ }^{10}$ A.I. Frenkel, J. Synchrotron Radiat. 6, 293 (1999).

${ }^{11}$ J.C. Cezar, H.C.N. Tolentino, and M. Knobel, Phys. Rev. B 68, 054404 (2003).

${ }^{12}$ A. Muñoz-Martín, M. Vila, C. Prieto, C. Ocal, and J.L. Martínez, Vacuum 67, 583 (2002).

${ }^{13}$ A. Muñoz-Martín, C. Prieto, C. Ocal, J.L. Martínez, and J. Colino, Surf. Sci. 482-485, 1095 (2001).

${ }^{14}$ J. Mimault, J.J. Faix, T. Girardeau, M. Jaouen, and G. Tourillon, Meas. Sci. Technol. 5, 482 (1994).

${ }^{15}$ K.V. Klementiev, "VIPER for Windows:" freeware at http://www.desy.de/ klmn/viper.html; K.V. Klementiev, J. Phys. D 34, 209 (2001).

${ }^{16}$ J.J. Rehr, Jpn. J. Appl. Phys., Part 1 32, 8 (1993).

${ }^{17}$ F. Jiménez-Villacorta, A. Muñoz-Martín, M. Vila, C. Prieto, and A. Traverse, Phys. Scr. (in press).

${ }^{18}$ R. Castañer, C. Prieto, A. De Andrés, J.L. Martínez, J.L. MartínezAlbertos, C. Ocal, and R. Miranda, J. Phys.: Condens. Matter 6, 4981 (1994).

${ }^{19}$ D. Chandesris, P. Le Fèvre, H. Magnan, A. Chaumin-Midoir, H. Jaffrès, F. Scheurer, and L. Barbier, J. Phys.: Condens. Matter 15, S657 (2003). 